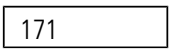

\section{NOX2-DERIVED REACTIVE OXYGEN SPECIES CAUSES VASCULAR DYSFUNCTION IN MURINE MODEL OF ENDOTHELIAL INSULIN SENSITIVITY AND ACTIVATION OF NRF2 TRANSCRIPTION FACTOR}

H Viswambharan, P Sukumar, M Gage, H Imrie, A Sengupta, J Turner, N Yuldasheva, A Skromna, S Galloway, J Smith, R Cubbon, S Wheatcroft, M Kearney University of Leeds

doi:10.1136/heartjnl-2013-304019.171

Introduction The escalating number of individuals suffering from Type 2 Diabetes is a significant healthcare burden, globally. A critical pathophysiological feature of type 2 diabetes is insulin resistance. It is well-established that insulin stimulates generation of the endothelium-derived anti-inflammatory/anti-atherosclerotic signalling radical, nitric oxide (NO). We investigated the hypothesis that increasing insulin sensitivity specifically, in the endothelium will lead to beneficial effects on NO bioavailability and vascular endothelial function. A novel transgenic mouse over-expressing Type A human Insulin Receptor (HIRECO) in the endothelium, driven by the Tie-2 promoter-enhancer was generated in order to explore the effects of increasing insulin signalling in the vascular bed.

Methods Various tissues and pulmonary endothelial cells from the HIRECO mice were analysed using RT-PCR to confirm significant levels of human insulin receptor mRNA, while protein expression was confirmed by western blotting. Lucigenin-enhanced chemiluminescence was used to measure superoxide anion levels while; vasomotor function was assessed in thoracic aortic rings mounted in an organ bath.

Results HIRECO mice demonstrated no significant morphological, metabolic phenotype or blood pressure abnormality compared to wild type (WT) littermates. Plasma insulin levels were similar following an overnight fast, but were decreased in the HIRECO after a glucose challenge. HIRECO mice exhibited significant endothelial dysfunction with a blunted response to acetylcholine (Emax, WT vs. HIRECO: $84 \pm 3 \%$ vs. $68 \pm 3 \%$ respectively, $p<0.05)$. The impaired aortic response to acetylcholine was normalized by the NADPH oxidase inhibitor peptide, gp91ds-tat, (Emax: 93 $\pm 5 \%$; $\mathrm{p}<0.05$ ), as well as the superoxide dismutase mimetic, MnTmPyP. HIRECO mice had a 1.65-fold increase in the level of superoxide anion production compared to WT. Basal NO bioactivity was decreased in HIRECO compared to WT littermates (Emax upon exposure to eNOS inhibitor, L-NAME in phenylephrine-constricted aorta, WT vs. HIRECO: $144 \pm 27.9 \%$ vs. $32 \pm 33 \%, n=5, p<0.05$ ). However, basal eNOS and Akt phosphorylation in isolated endothelial cells of HIRECO mice was enhanced 1.56 fold compared to WT littermates. Additionally, increased expressions of $\mathrm{NADPH}$ oxidase isoform, Nox2 and redox-sensitive transcription factor, Nrf2 were detected in HIRECO endothelial cells.
Conclusions/Implications These data clearly suggest that enhanced oxidative stress in a novel murine model of increased endothelial insulin signalling, leads to reduced bioavailability of nitric oxide and vascular dysfunction. These data also demonstrate for the first time, that increased insulin sensitivity in the endothelium, increases the generation of free radical generation and reduces $\mathrm{NO}$ bioavailability. 